\title{
A class function on the mapping class group of an orientable surface and the Meyer cocycle
}

\author{
MASATOSHi SATO
}

\begin{abstract}
In this paper we define a $\mathbf{Q} \mathbf{P}^{1}$-valued class function on the mapping class group $\mathcal{M}_{g, 2}$ of a surface $\Sigma_{g, 2}$ of genus $g$ with two boundary components. Let $E$ be a $\Sigma_{g, 2}$-bundle over a pair of pants $P$. Gluing to $E$ the product of an annulus and $P$ along the boundaries of each fiber, we obtain a closed surface bundle over $P$. We have another closed surface bundle by gluing to $E$ the product of $P$ and two disks. The sign of our class function cobounds the 2-cocycle on $\mathcal{M}_{g, 2}$ defined by the difference of the signature of these two surface bundles over $P$.
\end{abstract}

\section{Introduction}

Let $\Sigma_{g, r}$ be a compact oriented surface of genus $g$ with $r$ boundary components. The mapping class group $\mathcal{M}_{g, r}$ is $\pi_{0} \operatorname{Diff}_{+}\left(\Sigma_{g, r}, \partial \Sigma_{g, r}\right)$ where Diff $+\left(\Sigma_{g, r}, \partial \Sigma_{g, r}\right)$ is the group of orientation preserving diffeomorphisms of $\Sigma_{g, r}$ which restrict to the identity on the boundary $\partial \Sigma_{g, r}$. We simply denote $\Sigma_{g}:=\Sigma_{g, 0}$ and $\mathcal{M}_{g}:=\mathcal{M}_{g, 0}$. Harer [4] proved that

$$
H^{2}\left(\mathcal{M}_{g, r} ; \mathbf{Z}\right) \cong \mathbf{Z} \quad g \geq 3, r \geq 0,
$$

see also Korkmaz and Stipsicz [8].

Meyer [9] defined a cocycle $\tau_{g} \in Z^{2}\left(\mathcal{M}_{g} ; \mathbf{Z}\right)(g \geq 0)$ called the Meyer cocycle which represents four times generator of the second cohomology class when $g \geq 3$. Let $D_{1}, D_{2}$, and $D_{3}$ be mutually disjoint disks in $S^{2}$, and Int $D_{i}$ the interior of $D_{i}$ for $i=1,2,3$. We denote by $P:=S^{2}-\amalg_{i=1}^{3}$ Int $D_{i}$ the pair of pants, and $\alpha, \beta, \gamma \in \pi_{1}(P)$ be the homotopy classes as shown in Figure 1. We consider a $\Sigma_{g, r}$-bundle $E_{g, r}^{\varphi, \psi}$ on the pair of pants $P$ which has monodromies $\varphi, \psi,(\psi \varphi)^{-1} \in \mathcal{M}_{g, r}$ along $\alpha, \beta$, $\gamma \in \pi_{1}(P)$. The diffeomorphism type of $E_{g, r}^{\varphi, \psi}$ does not depend on the choice of representatives in the mapping classes $\varphi$ and $\psi$. Since $E_{g, r}^{\varphi, \psi}$ is the oriented fiber 


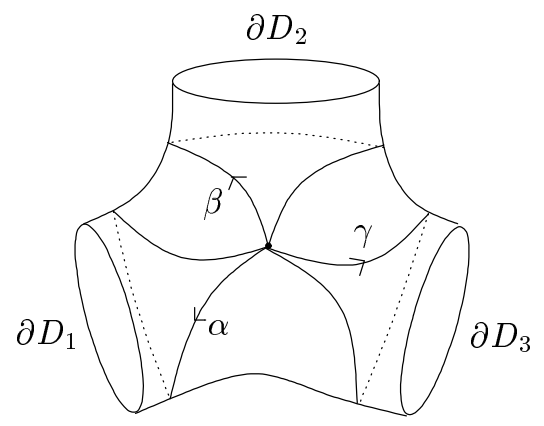

Figure 1

bundle, it has the canonical orientation comes from that of $\Sigma_{g, r}$ and $P$. The Meyer cocycle is defined by

$$
\begin{aligned}
\tau_{g}: \mathcal{M}_{g} \times \mathcal{M}_{g} & \rightarrow \quad \mathbf{Z} \\
(\varphi, \psi) & \mapsto \operatorname{Sign} E_{g}^{\varphi, \psi}
\end{aligned}
$$

where $\operatorname{Sign} E_{g}^{\varphi, \psi}$ is the signature of the compact oriented 4-manifold $E_{g}^{\varphi, \psi}$. For $k>0$, it is known as Novikov additivity that when two compact oriented $4 k$-manifolds are glued by an orientation reversing diffeomorphism of their boundaries, the signature of their union is the sum of their signature. When a pants decomposition of a closed oriented 2-manifold is given, the signature of a $\Sigma_{g}$-bundle on the 2-manifold is the sum of the signature of the $\Sigma_{g}$-bundles restricted to each pair of pants. Therefore, it is important to study the Meyer cocycle to calculate the signature of compact 4-manifolds. For $g=1,2$ the Meyer cocycle $\tau_{g}$ is a coboundary, and the cobounding function of this cocycle is calculated by several authors, for instance, Meyer [9], Atiyah [1], Kasagawa [6] and Iida [5]. The Meyer cocycle is not a coboundary if genus $g \geq 3$, but the cocycle can be a coboundary when it is restricted to some subgroups. For example, on the subgroup called the hyperelliptic mapping class group, the cobounding function is calculated by Endo [2] and Morifuji [11].

Let $I$ be the unit interval $[0,1] \subset \mathbf{R}$. By sewing a pair of disks onto the surface $\Sigma_{g, 2}$ along the boundary, we have $\Sigma_{g}$. For $h \in \operatorname{Diff}_{+}\left(\Sigma_{g, 2}, \partial \Sigma_{g, 2}\right)$, if we extend $h$ by the identity on the pair of disks, we have a self-diffeomorphism of $\Sigma_{g}$. We denote it by $h \cup i d_{\amalg_{i=1}^{2} D^{2}}$. By sewing an annulus $S^{1} \times I$ onto the surface $\Sigma_{g, 2}$ along the boundary, we have $\Sigma_{g+1}$. In the same way, if we extend $h \in \operatorname{Diff}_{+}\left(\Sigma_{g, 2}, \partial \Sigma_{g, 2}\right)$ by the identity on the annulus, we have a self-diffeomorphism $h \cup i d_{S^{1} \times I}$. 
Define the induced homomorphism on the mapping class group by

$$
\begin{aligned}
\theta: \mathcal{M}_{g, 2} & \rightarrow \quad \mathcal{M}_{g} \\
{[h] } & \mapsto\left[h \cup i d_{\amalg_{i=1}^{2} D^{2}}\right]
\end{aligned}
$$

and

$$
\begin{aligned}
\eta: \mathcal{M}_{g, 2} & \rightarrow \mathcal{M}_{g+1,0} . \\
{[h] } & \mapsto\left[h \cup i d_{S^{1} \times I}\right] .
\end{aligned}
$$

Harer [3; 4] shows that $\theta$ and $\eta$ induce an isomorphism on the second homology classes when genus $g \geq 5$, so that $\tilde{\tau}_{g}=\eta^{*} \tau_{g+1}-\theta^{*} \tau_{g}$ is a coboundary. Powell [12] proved that the first homology group $H_{1}\left(\mathcal{M}_{g, r} ; \mathbf{Z}\right)$ is trivial for $g \geq 3$ and $r \geq 0$, so by the universal coefficient theorem, it follows that the cobounding function of $\tilde{\tau}_{g}$ is unique.

In this paper we define a $\mathbf{Q} \mathbf{P}^{1}$-valued class function $m$ on the mapping class group $\mathcal{M}_{g, 2}$ in an explicit way by using information of the first homology group of a mapping torus of $[h] \in \mathcal{M}_{g, 2}$. For $[p: q] \in \mathbf{Q P}^{1}$, we define the sign of $[p: q]$ by sign ([p: $q]):=\operatorname{sign}(p q)$. We prove that the sign of the function $m$ cobounds the cocycle $\tilde{\tau}_{g}=\eta^{*} \tau_{g+1}-\theta^{*} \tau_{g}$. In particular, it turns out that the cocycle $\tilde{\tau}_{g}$ is coboundary for any $g \geq 0$.

This function makes a little bit easy to evaluate the Meyer cocycle on the subgroups consists of mapping classes that fix a curve on the surface. For example, consider the case $g=1,2$. We denote by $\phi_{1}$ and $\phi_{2}$ the cobounding functions of $\tau_{1}$ and $\tau_{2}$. Since $H_{1}\left(\mathcal{M}_{g, 2} ; \mathbf{Q}\right)=0$, the equation $\eta^{*} \tau_{g+1}=\theta^{*} \tau_{g}+\delta m$ means $\eta^{*} \phi_{g+1}=\theta^{*} \phi_{g}+m$ for $g=1,2$. In particular, the function $\phi_{1}$ is described explicitly in Meyer [9]. Therefore, our function $m$ helps to describe the cobounding function of the Meyer cocycle for genus 2 and 3 on the subgroup.

In Section 2, we construct a class function $m$, prove some properties of this function, and calculate the image of the function. In Section 3, we prove that the sign of this function cobounds the difference $\tilde{\tau}_{g}=\eta^{*} \tau_{g+1}-\theta^{*} \tau_{g}$. By the definition of the Meyer cocycle $\tau_{g}, \tilde{\tau}_{g}(\varphi, \psi)$ is just the difference Sign $E_{g+1}^{\eta(\varphi), \eta(\psi)}-\operatorname{Sign} E_{g}^{\theta(\varphi), \theta(\psi)}$, so that we calculate the difference by using the sign of the function $m$. Moreover we compute the other differences of signature $\operatorname{Sign}\left(E_{g, 2}^{\varphi, \psi}\right)-\operatorname{Sign}\left(E_{g}^{\theta(\varphi), \theta(\psi)}\right)$ and $\operatorname{Sign}\left(E_{g+1}^{\eta(\varphi), \eta(\psi)}\right)-\operatorname{Sign}\left(E_{g, 2}^{\varphi, \psi}\right)$ by the function $m$.

\section{Class function $m: \mathcal{M}_{g, 2} \rightarrow \mathbf{Q P}^{1}$}

In this section we define the class function on the mapping class group $\mathcal{M}_{g, 2}$ stated in the introduction and describe some properties of the function including the nontriviality. 
For $[p: q],[r: s] \in \mathbf{Q} \mathbf{P}^{1}$, we define an addition in $\mathbf{Q} \mathbf{P}^{1}$ by

$$
[p: q]+[r: s]= \begin{cases}{[p r: p s+q r],} & \text { if }[p: q] \neq[0: 1] \text { or }[r: s] \neq[0: 1] \\ {[0: 1],} & \text { if }[p: q]=[r: s]=[0: 1] .\end{cases}
$$

The projective line $\mathbf{Q} \mathbf{P}^{1}$ forms an additive monoid under this operation with [1:0] the zero element.

In this section, all (co)homology groups are with $\mathbf{Q}$ coefficients.

\subsection{Construction of the class function}

Before constructing the function, we prepare a fact about homology groups of compact 3-manifolds. Let $Y$ be a compact oriented connected 3-manifold with boundary $\partial Y$ and $i: \partial Y \hookrightarrow Y$ the inclusion map. Consider the commutative diagram

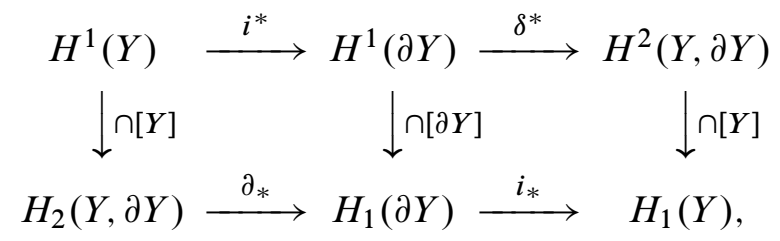

where the upper and lower rows are the exact sequences of a pair $(Y, \partial Y)$, and the vertical maps are the cap products with the (relative) fundamental classes of $Y$ and $\partial Y$. By the diagram and Poincaré Duality, it follows that the image of $i^{*}$ is just its own annihilator with respect to the cup product of $H^{1}(\partial Y)$

$$
\operatorname{Im} i^{*}=\operatorname{Ann}\left(\operatorname{Im} i^{*}\right) .
$$

In particular, we have

$$
\operatorname{dim} \operatorname{Ker} i_{*}=\operatorname{dim} \operatorname{Im} i^{*}=\frac{1}{2} \operatorname{dim} H_{1}(\partial Y) .
$$

We define the mapping torus of $\varphi=[h] \in \mathcal{M}_{g, r}$ by

$$
X^{\varphi}:=\Sigma_{g, r} \times I / \sim, \quad(x, 1) \sim(h(x), 0),
$$

and $\pi: X^{\varphi} \rightarrow I / \partial I=S^{1}$ by the projection $\pi([x, t])=[t]$, where $[x, t] \in X^{\varphi}$ is the equivalent class of $(x, t) \in \Sigma_{g, r} \times I$, and $[t] \in I / \partial I=S^{1}$ the equivalent class of $t \in I$.

The diffeomorphism type of the mapping torus $X^{\varphi}$ does not depend on the choice of the representative $h$. We fix the orientation on $X^{\varphi}$ given by the product orientation on $\Sigma_{g, r} \times I$. Let $i_{\varphi}: \partial X^{\varphi} \hookrightarrow X^{\varphi}$ be the inclusion map. In this subsection we denote $\Sigma:=\Sigma_{g, 2}$, and if we fix $\varphi \in \mathcal{M}_{g, 2}$, then we write simply $X:=X^{\varphi}$ and $i:=i_{\varphi}$. Let $S_{1}$ and $S_{2}$ be the two boundary components of $\Sigma$, and $\left[S_{k}\right](k=1,2)$ the image 
under the inclusion homomorphism $H_{1}\left(S_{k}\right) \rightarrow H_{1}(\Sigma)$ of the fundamental homology class.

We consider $\Sigma$ as a subspace of $X$ by the embedding $\iota: \Sigma \hookrightarrow X$ by $x \mapsto[x, 0]$. We choose points $p_{1} \in S_{1}, p_{2} \in S_{2}$, and $p \in S^{1}$, and orientation-preserving homeomorphisms $\iota_{1}: S^{1} \rightarrow S_{1}$ and $\iota_{2}: S^{1} \rightarrow S_{2}$. We define singular chains $f_{k}: I \rightarrow$ $\left(S_{1} \amalg S_{2}\right) \times S^{1}=\partial X(k=1,2,3,4)$ by

$f_{1}(t)=\left(\iota_{1}(t), p\right), f_{2}(t)=\left(\iota_{2}(t), p\right), f_{3}(t)=\left(p_{1}, t\right)$ and $f_{4}(t)=\left(p_{2}, t\right)$ respectively.

Let $e_{k} \in H_{1}(\partial X)$ be the homology class of $f_{k}(k=1,2,3,4)$. Then the set $\left\{e_{1}, e_{2}, e_{3}, e_{4}\right\}$ forms a basis for $H_{1}(\partial X)$, and the intersection number

$$
e_{i} \cdot e_{j}= \begin{cases}1 & \text { if } j=i+2 \\ 0 & \text { otherwise }\end{cases}
$$

for $i=1,2$ and $j=3,4$. Now we describe the kernel of the homomorphism $i_{*}: H_{1}(\partial X) \rightarrow H_{1}(X)$. Since $e_{1}$ and $e_{2}$ lie in the kernel of $\left(\left.\pi\right|_{\partial X}\right)_{*}$ and $\pi_{*}\left(e_{3}\right)=$ $\pi_{*}\left(e_{4}\right)=\left[S^{1}\right] \in H_{1}\left(S^{1}\right)$, we have

$$
\operatorname{Ker} i_{*} \subset \operatorname{Ker}\left(\pi_{*} i_{*}\right)=\mathbf{Q} e_{1} \oplus \mathbf{Q} e_{2} \oplus \mathbf{Q}\left(e_{3}-e_{4}\right) .
$$

By the definition of the map $f_{k},\left(i \circ f_{k}\right)_{*}\left[S^{1}\right]=\iota_{*}\left[S_{k}\right]$, and so $i_{*}\left(e_{1}+e_{2}\right)=\iota_{*}\left(\left[S_{1}\right]+\right.$ $\left.\left[S_{2}\right]\right) \in H_{1}(X)$. Since $S_{1} \cup S_{2}$ is the boundary of $\Sigma$, we have $\left[S_{1}\right]+\left[S_{2}\right]=0 \in H_{1}(\Sigma)$. Hence

$$
\mathbf{Q}\left(e_{1}+e_{2}\right) \subset \operatorname{Ker} i_{*} .
$$

As we saw at the beginning of this subsection, $\operatorname{dim} \operatorname{Ker} i_{*}=\frac{1}{2} \operatorname{dim} H_{1}(\partial X)=2$. It follows that $\operatorname{Ker} i_{*}=\mathbf{Q}\left(e_{1}+e_{2}\right) \oplus \mathbf{Q}\left(p\left(e_{3}-e_{4}\right)+q e_{1}\right)$ for some $p, q \in \mathbf{Q}$. Now we can define a class function.

Definition 2.1 For $\varphi \in \mathcal{M}_{g, 2}$, we take $p, q \in \mathbf{Q}$ such that $\operatorname{Ker} i_{\varphi *}=\mathbf{Q}\left(e_{1}+e_{2}\right) \oplus$ $\mathbf{Q}\left(p\left(e_{3}-e_{4}\right)+q e_{1}\right)$.

We define $m: \mathcal{M}_{g, 2} \rightarrow \mathbf{Q P}^{\mathbf{1}}$ by $m(\varphi)=[p: q]$.

Lemma 2.2 For $\varphi, \psi \in \mathcal{M}_{g, 2}$,

$$
m\left(\psi \varphi \psi^{-1}\right)=m(\varphi)
$$

Proof Define $\Psi: X^{\varphi} \rightarrow X^{\psi \varphi \psi^{-1}}$ by $\Psi(x, t)=(\psi(x), t)$. Then $\Psi$ maps $e_{i}$ as defined in $H_{1}\left(X^{\varphi}\right)$ to the corresponding $e_{i}$ as defined in $H_{1}\left(X^{\psi \varphi \psi^{-1}}\right)$, and the 
following diagram commutes

$$
\begin{array}{ccc}
H_{1}\left(\partial X^{\varphi}\right) & \stackrel{i_{\varphi *}}{\longrightarrow} & H_{1}\left(X^{\varphi}\right) \\
\downarrow \Psi_{*} & & \downarrow \Psi_{*} \\
H_{1}\left(\partial X^{\psi \varphi \psi^{-1}}\right) \stackrel{i_{\psi \varphi \psi^{-1}} *}{\longrightarrow} & H_{1}\left(X^{\psi \varphi \psi^{-1}}\right) .
\end{array}
$$

As we see from the diagram, $\Psi_{*}$ gives the natural isomorphism between the kernels $\operatorname{Ker}\left(H_{1}\left(\partial X^{\varphi}\right) \rightarrow H_{1}\left(X^{\varphi}\right)\right)$ and $\operatorname{Ker}\left(H_{1}\left(\partial X^{\psi \varphi \psi^{-1}}\right) \rightarrow H_{1}\left(X^{\psi \varphi \psi^{-1}}\right)\right)$. Hence we have $m\left(\psi \varphi \psi^{-1}\right)=m(\varphi)$.

\subsection{Some properties and the nontriviality of the class function}

By the Serre spectral sequence of the $\Sigma$-bundle $\pi: X \rightarrow S^{1}$, we have the exact sequence

$$
0 \longrightarrow \operatorname{Coker}\left(\varphi_{*}-1\right) \stackrel{\iota_{*}}{\longrightarrow} H_{1}(X) \stackrel{\pi_{*}}{\longrightarrow} H_{1}\left(S^{1}\right) \longrightarrow 0,
$$

where Coker $\left(\varphi_{*}-1\right)$ is the cokernel of the homomorphism $\varphi_{*}-1: H_{1}(\Sigma) \rightarrow H_{1}(\Sigma)$. Then we have a unique homomorphism $j_{\varphi}: \mathbf{Q} e_{1} \oplus \mathbf{Q} e_{2} \oplus \mathbf{Q}\left(e_{3}-e_{4}\right) \rightarrow \operatorname{Coker}\left(\varphi_{*}-1\right)$ such that the diagram with exact rows

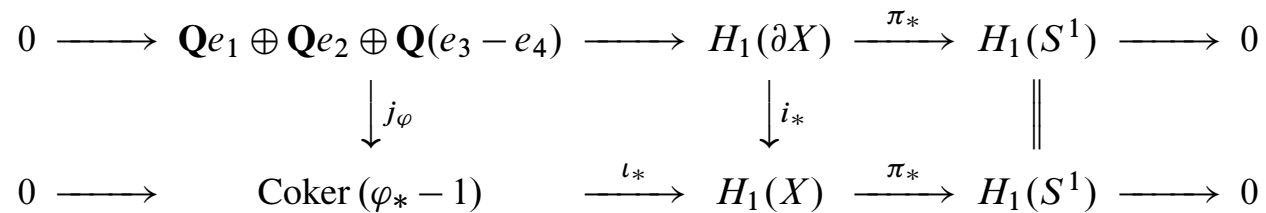

commutes. By the diagram, we have

$$
\begin{aligned}
& \operatorname{Ker} i_{*}=\operatorname{Ker} j_{\varphi} \text { and } \\
& j_{\varphi}\left(e_{1}\right)=-j_{\varphi}\left(e_{2}\right)=\left[S_{1}\right] \in \operatorname{Coker}\left(\varphi_{*}-1\right) .
\end{aligned}
$$

Now we introduce a cochain $\omega_{l} \in C^{1}\left(\mathcal{M}_{g, 2} ; H_{1}(\Sigma)\right)$ defined by Kawazumi [7]. On the fiber $\Sigma=\pi^{-1}(0) \subset X$, pick a path $l$ such that $l(0) \in S_{2}$ and $l(1) \in S_{1}$. Define $\omega_{l}$ by

$$
\omega_{l}(\varphi):=[\varphi(l)-l] \in H_{1}(\Sigma) .
$$

Then we have the following lemma.

\section{Lemma 2.3}

$$
j_{\varphi}\left(e_{3}-e_{4}\right)=\left[\omega_{l}(\varphi)\right] \in \operatorname{Coker}\left(\varphi_{*}-1\right) .
$$


Proof Define a 2-chain $L: I \times I \rightarrow X$ by $L(s, t)=[l(s), t]$. Its boundary is given by $-i_{*}\left(e_{3}\right)+\varphi(l)+i_{*}\left(e_{4}\right)-l \in B_{1}(X)$. Hence,

$$
i_{*}\left(e_{3}-e_{4}\right)=\iota_{*}([\varphi(l)-l]) \in H_{1}(X)
$$

Since $\iota_{*}$ is injective, the lemma follows.

From the lemma, we see the homology class $\left[\omega_{l}(\varphi)\right] \in \operatorname{Coker}\left(\varphi_{*}-1\right)$ is independent of the choice of the path $l$. If $\omega_{l}(\varphi)=0$, then $j_{\varphi}\left(e_{3}-e_{4}\right)=0$.

Remark 2.4 If there exists a path $l$ from a point in $S_{2}$ to a point in $S_{1}$ which has no common point with the support of a representative of $\varphi \in \mathcal{M}_{g, 2}$, then $m(\varphi)=[1: 0]$. In particular, $m(i d)=[1: 0]$, the zero element of the monoid $\mathbf{Q P}{ }^{1}$.

Define the subgroups $\mathcal{I}^{\prime}:=\operatorname{Ker}\left(\mathcal{M}_{g, 2} \rightarrow \operatorname{Aut}\left(H_{1}\left(\Sigma_{g, 2} ; \mathbf{Z}\right)\right)\right.$ and $\mathcal{I}:=\operatorname{Ker}\left(\mathcal{M}_{g, 2} \rightarrow\right.$ Aut $\left(H_{1}\left(\Sigma_{g, 2}, \partial \Sigma_{g, 2} ; \mathbf{Z}\right)\right)$. For $\varphi \in \mathcal{I}^{\prime}, m(\varphi)=[p: q]$ means $p(\varphi(l)-l)+q e_{1}=$ $0 \in H_{1}\left(\Sigma_{g, 2} ; \mathbf{Z}\right)$. This shows that $m$ is homomorphic on $\mathcal{I}^{\prime}$. For $\varphi \in \mathcal{I}, \omega(\varphi)=0 \in$ $H_{1}\left(\Sigma_{g, 2} ; \mathbf{Z}\right)$. This shows that $m(\varphi)=[1: 0]$ for all $\varphi \in \mathcal{I}$.

Remark 2.5 The restriction of $m$ on $\mathcal{I}$ is trivial, and the restriction of $m$ on $\mathcal{I}^{\prime}$ is a nontrivial monoid homomorphism.

At the beginning of this section, we defined the commutative monoid structure on $\mathbf{Q P} \mathbf{P}^{\mathbf{1}}$. So integral multiples of $m(\varphi)$ are well-defined.

Proposition 2.6 If $\varphi \in \mathcal{M}_{g, 2}$ and $k \in \mathbf{Z}$, then

$$
m\left(\varphi^{k}\right)=k m(\varphi)
$$

Proof The proposition is trivial for $k=0$ and $k=1$. Assume $k \geq 2$.

Let $m(\varphi)=[p: q]$. By the definition of $j_{\varphi}, p j_{\varphi}\left(e_{3}-e_{4}\right)=-q\left[S_{1}\right] \in \operatorname{Coker}\left(\varphi_{*}-1\right)$. Hence, there exists $v \in H_{1}(\Sigma)$ such that

$$
p[\varphi(l)-l]=-q\left[S_{1}\right]+\left(\varphi_{*}-1\right) v \in H_{1}(\Sigma) .
$$

Apply $\varphi^{i}(i=0,1, \ldots, k-1)$ to the both sides of the equation and sum over $i$. Then

$$
\sum_{i=0}^{k-1} p\left[\varphi^{i+1}(l)-\varphi^{i}(l)\right]=\sum_{i=0}^{k-1}\left\{-q\left[S_{1}\right]+\left(\varphi_{*}^{i+1}(v)-\varphi_{*}^{i}(v)\right)\right\},
$$

that is

$$
p\left[\varphi^{k}(l)-l\right]=-k q\left[S_{1}\right]+\left(\varphi_{*}^{k}-1\right) v .
$$


Hence, $m\left(\varphi^{k}\right)=[p: k q]=k m(\varphi)$ for $k \geq 0$.

By applying $\varphi^{-1}$ to the equation $p[\varphi(l)-l]=-q\left[S_{1}\right]+\left(\varphi_{*}-1\right) v$, we have

$$
p\left[\varphi^{-1}(l)-l\right]=q\left[S_{1}\right]+\left(\varphi_{*}^{-1}-1\right) v \in H_{1}(\Sigma) .
$$

Hence, $m\left(\varphi^{-1}\right)=[p:-q]=-m(\varphi)$. Since $m\left(\varphi^{-k}\right)=-m\left(\varphi^{k}\right)=-k m(\varphi)$ for $k>0$, the proposition follows for the case $k<0$.

Now we compute the image of the function $m$. In particular, we see that $m$ is nontrivial.

Proposition 2.7 For $g \geq 1, m$ is surjective. For $g=0, \operatorname{Im}(m)=[1: \mathbf{Z}]$.

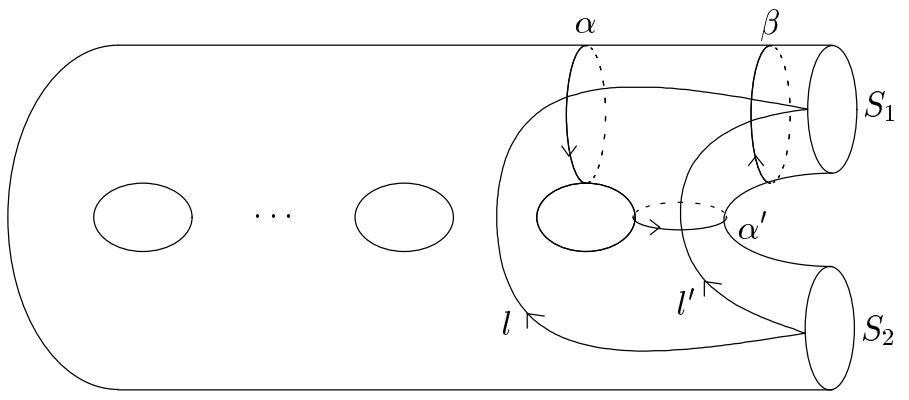

Figure 2

Proof Suppose $g \geq 1$. We choose oriented simple closed curves $\alpha, \alpha^{\prime}$, and $\beta$ and paths $l$ and $l^{\prime}$ as shown in Figure 2. We denote the Dehn twists along a simple closed curve $C \subset \Sigma$ by $t_{C}$, and the homology class of $C$ by $[C]$. Then $[\alpha]+\left[\alpha^{\prime}\right]+[\beta]=0 \in H_{1}(\Sigma)$ since they bound a $2-$ chain. For $p \in \mathbf{Z}$, if we denote $\varphi:=t_{\alpha}^{p} t_{\alpha^{\prime}} t_{\beta}^{-1}$, then

$$
\begin{aligned}
j_{\varphi}\left((p+1)\left(e_{3}-e_{4}\right)\right) & =\omega_{l}(\varphi)+p \omega_{l^{\prime}}(\varphi) \\
& =\left[\left(t_{\alpha}^{p} t_{\alpha^{\prime}} t_{\beta}^{-1}\right)(l)-l\right]+p\left[\left(t_{\alpha}^{p} t_{\alpha^{\prime}} t_{\beta}^{-1}\right)\left(l^{\prime}\right)-l^{\prime}\right] \\
& =p\left([\alpha]+\left[\alpha^{\prime}\right]+[\beta]\right)+[\beta]=[\beta]=\left[S_{1}\right] .
\end{aligned}
$$

Hence, $j_{\varphi}\left((p+1)\left(e_{3}-e_{4}\right)-e_{1}\right)=0$, so that

$$
m(\varphi)=[p+1:-1] .
$$

By Proposition 2.6, we have

$$
m\left(\varphi^{-q}\right)=-q[p+1:-1]=\left\{\begin{array}{ll}
{[p+1: q],} & \text { if } p \neq-1 \\
{[0: 1],} & \text { if } p=-1 .
\end{array} \quad(q \in \mathbf{Z})\right.
$$


Since $p$ and $q$ can run over all integers, we see $m$ is surjective for $g \geq 1$. For $g=0, \mathcal{M}_{0,2}$ is the infinite cyclic group generated by $t_{\beta}$. Since $m\left(t_{\beta}^{-q}\right)=[1: q]$, we have $\operatorname{Im}(m)=[1: \mathbf{Z}]$.

\section{The difference of two Meyer cocycles $\eta^{*} \tau_{g+1}$ and $\theta^{*} \tau_{g}$}

In this section (co)homology groups are with $\mathbf{Z}$ coefficient unless specified.

Let $g \geq 0$ be a positive integer. In the introduction, we defined the homomorphisms $\eta: \mathcal{M}_{g, 2} \rightarrow \mathcal{M}_{g+1,0}$ and $\theta: \mathcal{M}_{g, 2} \rightarrow \mathcal{M}_{g}$ to be the induced maps by sewing a pair of disks and by sewing an annulus onto the surface $\Sigma_{g, 2}$ along their boundaries respectively. We denote the Meyer cocycle on the mapping class group of genus $g$ closed orientable surface $\mathcal{M}_{g}$ by $\tau_{g} \in Z^{2}\left(\mathcal{M}_{g}\right)$ and define $\tilde{\tau}_{g} \in Z^{2}\left(\mathcal{M}_{g, 2}\right)$ to be the difference between the Meyer cocycles

$$
\tilde{\tau}_{g}:=\eta^{*} \tau_{g+1}-\theta^{*} \tau_{g} .
$$

Let $P:=S^{2}-\amalg_{i=1}^{3} D^{2}$. In this section, we prove the main theorem and calculate the changes of signature associated with sewing a pair of trivial disk bundles $P \times \amalg_{i=1}^{2} D^{2}$ and sewing a trivial annulus bundles $P \times\left(S^{1} \times I\right)$ onto $\Sigma_{g, 2}$-bundle on the pair of pants $P$ along their boundaries. To state the main theorem, we define the sign of $[p: q] \in \mathbf{Q P}^{\mathbf{1}}$ by

$$
\operatorname{sign}([p: q]):=\operatorname{sign}(p q)= \begin{cases}1 & \text { if } p q>0, \\ 0 & \text { if } p q=0, \\ -1 & \text { if } p q<0 .\end{cases}
$$

Theorem 3.1 For $\varphi, \psi \in \mathcal{M}_{g, 2}$, we define

$$
\widetilde{\phi}_{g}(\varphi):=\operatorname{sign}(m(\varphi)) \text {. }
$$

Then $\tilde{\phi}_{g}$ cobounds the difference $\tilde{\tau}_{g}$ between the Meyer cocycles $\eta^{*} \tau_{g+1}$ and $\theta^{*} \tau_{g}$

$$
\begin{aligned}
\tilde{\tau}_{g}(\varphi, \psi) & =\delta \tilde{\phi}_{g}(\varphi, \psi) \\
& =\operatorname{sign}(m(\varphi))+\operatorname{sign}(m(\psi))+\operatorname{sign}\left(m\left((\varphi \psi)^{-1}\right)\right) .
\end{aligned}
$$

Remark 3.2 Let $k$ be an integer. By Lemma 2.2 and Proposition 2.6, $\widetilde{\phi}_{g}$ has the properties

$$
\begin{aligned}
\tilde{\phi}_{g}\left(\psi \varphi \psi^{-1}\right) & =\tilde{\phi}_{g}(\varphi) \text { and } \\
\widetilde{\phi}_{g}\left(\varphi^{k}\right) & =\operatorname{sign}(k) \tilde{\phi}_{g}(\varphi)
\end{aligned}
$$


for any $g \geq 0$.

\subsection{Proof of Main Theorem}

In this subsection we prove Theorem 3.1.

In the introduction, we defined compact oriented 4-manifold $E_{g, r}^{\varphi, \psi}$ as a $\Sigma_{g, r}$-bundle on the pair of pants $P$ which has monodromies $\varphi, \psi$, and $(\psi \varphi)^{-1} \in \mathcal{M}_{g, r}$ along $\alpha, \beta$, and $\gamma \in \pi_{1}(P)$ respectively, and in Section 2.1, we defined compact oriented 3 -manifold $X_{g, r}^{\varphi}$ by the mapping torus of $\Sigma_{g, r} \times I / \sim$ where $(x, 1) \sim(h(x), 0)$ for $\varphi=[h] \in \mathcal{M}_{g, r}$.

Gluing to $E_{g, 2}^{\eta(\varphi), \eta(\psi)}$ the trivial annulus bundle on $P$ along the boundaries of each fiber, we obtain

$$
E_{g+1}^{\eta(\varphi), \eta(\psi)}=E_{g, 2}^{\varphi, \psi} \cup\left(-S^{1} \times I \times P\right) .
$$

Similarly, glue to $X_{g, 2}^{\eta(\varphi)}$ the trivial annulus bundle on $S^{1}$. Then we have

$$
X_{g+1}^{\eta(\varphi)}=X_{g, 2}^{\varphi} \cup\left(-S^{1} \times I \times S^{1}\right) .
$$

Define

$$
\begin{aligned}
G: \quad \partial D^{2} \times I & \rightarrow\{1\} \times S^{1} \times I . \\
(x, t) & \mapsto\left(1, x, \frac{1+t}{3}\right) .
\end{aligned}
$$

By the map $G$, we can glue $D^{2} \times I$ to $I \times S^{1} \times I$ as shown in Figure 3 .

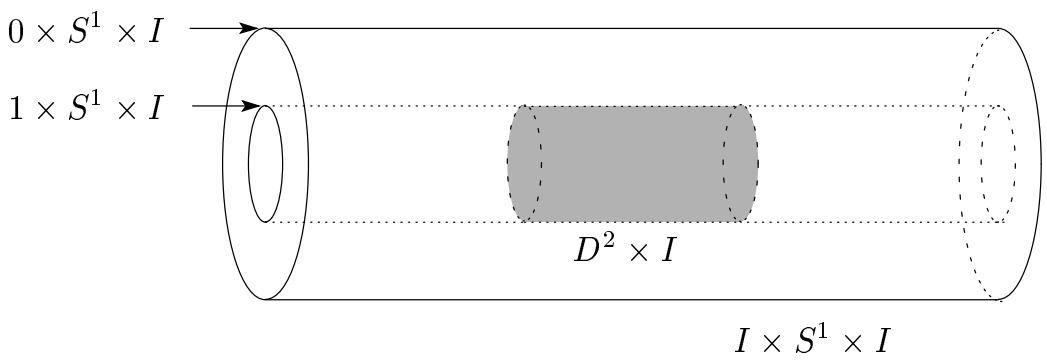

Figure 3: Gluing map $G$

Glue $D^{2} \times I \times P$ to $I \times E_{g+1}^{\eta(\varphi), \eta(\psi)}=\left(I \times E_{g, 2}^{\varphi, \psi}\right) \cup\left(-I \times S^{1} \times I \times P\right)$ with the gluing map $G \times i d_{P}: \partial D^{2} \times I \times P \rightarrow\{1\} \times S^{1} \times I \times P$. In the same way, glue $D^{2} \times I \times S^{1}$ 
to $I \times X_{g+1}^{\eta(\varphi)}=\left(I \times X_{g, 2}^{\varphi}\right) \cup\left(-I \times S^{1} \times I \times S^{1}\right)$ with $G \times i d_{S^{1}} \partial D^{2} \times I \times S^{1} \rightarrow$ $\{1\} \times S^{1} \times I \times S^{1}$. Namely, we construct two manifolds

$$
\widetilde{E}^{\varphi, \psi}:=\left(I \times E_{g+1}^{\eta(\varphi), \eta(\psi)}\right) \cup_{G \times i d_{P}}\left(D^{2} \times I \times P\right)
$$

and

$$
\tilde{X}^{\varphi}:=\left(I \times X_{g+1}^{\eta(\varphi)}\right) \cup_{G \times i d_{S^{1}}}\left(D^{2} \times I \times S^{1}\right) .
$$

Fix the orientations of these manifolds induced from the product orientations of $I \times$ $E_{g+1}^{\eta(\varphi), \eta(\psi)}$ and $I \times X_{g+1}^{\eta(\varphi)}$. To prove main theorem, it suffices to prove Lemma 3.3 and Lemma 3.4 below.

\section{Lemma 3.3}

$\left(\eta^{*} \tau_{g+1}-\theta^{*} \tau_{g}\right)(\varphi, \psi)=\operatorname{Sign} \tilde{X}^{\varphi}+\operatorname{Sign} \tilde{X}^{\psi}+\operatorname{Sign} \tilde{X}^{(\varphi \psi)^{-1}}$ for $\varphi, \psi \in \mathcal{M}_{g, 2}, g \geq 0$

\section{Lemma 3.4}

$$
\operatorname{Sign} \tilde{X}^{\varphi}=\operatorname{sign}(m(\varphi)) \text { for } \varphi \in \mathcal{M}_{g, 2}, \quad g \geq 0 .
$$

Proof of Lemma 3.3 Note that

$$
\tilde{X}^{\varphi}=\left.\widetilde{E}^{\varphi, \psi}\right|_{\partial D_{1}}
$$

Then we can see

$$
\begin{aligned}
\partial \widetilde{E}^{\varphi, \psi} & =\left(\left.\left.\left.\widetilde{E}^{\varphi, \psi}\right|_{\partial D_{1}} \cup \widetilde{E}^{\varphi, \psi}\right|_{\partial D_{2}} \cup \widetilde{E}^{\varphi, \psi}\right|_{\partial D_{3}}\right) \cup E_{g}^{\theta(\varphi), \theta(\psi)} \cup-E_{g+1}^{\eta(\varphi), \eta(\psi)} \\
& =\left(\tilde{X}^{\varphi} \cup \tilde{X}^{\psi} \cup \tilde{X}^{(\psi \varphi)^{-1}}\right) \cup E_{g}^{\theta(\varphi), \theta(\psi)} \cup-E_{g+1}^{\eta(\varphi), \eta(\psi)} .
\end{aligned}
$$

Since the Signature is a bordism invariant (for example, see Milnor and Stasheff [10, Lemma 17.3]), we have Sign $\partial \widetilde{E}^{\varphi, \psi}=0$. By Novikov Additivity, we see that

$$
\operatorname{Sign}\left(E_{g+1}^{\eta(\varphi), \eta(\psi)}\right)-\operatorname{Sign}\left(E_{g}^{\theta(\varphi), \theta(\psi)}\right)=\operatorname{Sign} \tilde{X}^{\varphi}+\operatorname{Sign} \tilde{X}^{\psi}+\operatorname{Sign} \tilde{X}^{(\psi \varphi)^{-1}} .
$$

Notice that $\tilde{X}^{(\psi \varphi)^{-1}}$ is diffeomorphic to $\tilde{X}^{(\varphi \psi)^{-1}}$, so that $\operatorname{Sign} \tilde{X}^{(\psi \varphi)^{-1}}=\operatorname{Sign} \tilde{X}^{(\varphi \psi)^{-1}}$. By the definition of the Meyer cocycle, we have

$$
\operatorname{Sign}\left(E_{g+1}^{\eta(\varphi), \eta(\psi)}\right)=\eta^{*} \tau_{g+1}(\varphi, \psi) \text {, and } \operatorname{Sign}\left(E_{g}^{\theta(\varphi), \theta(\psi)}\right)=\theta^{*} \tau_{g}(\varphi, \psi) .
$$

Define $\tilde{\phi}(\underset{\phi}{\varphi})=\operatorname{Sign}\left(\tilde{X}^{\varphi}\right)$; then we have $\delta \tilde{\phi}=\eta^{*} \tau_{g+1}-\theta^{*} \tau_{g}$. We get the cobounding function $\tilde{\phi}$. 
Proof of Lemma 3.4 Write simply $X:=X_{g+1}^{\eta(\varphi)}, X^{\prime}:=X_{g, 2}^{\varphi}$, and $Y:=\tilde{X}^{\varphi}=$ $(I \times X) \cup_{G \times i d_{S^{1}}}\left(D^{2} \times I \times S^{1}\right)$.

For $i=0,1$, define

$$
\begin{aligned}
j_{i}: X & \rightarrow I \times X \hookrightarrow Y, \\
x & \mapsto(i, x)
\end{aligned}
$$

where $I \times X \hookrightarrow Y$ is a natural embedding. We will prove there is a exact sequence

$$
H_{2}\left(X^{\prime}\right) \stackrel{j_{0 *}=j_{1 *}}{\longrightarrow} H_{2}(Y) \longrightarrow \operatorname{Ker}\left(H_{1}\left(\partial X^{\prime}\right) \rightarrow H_{1}\left(X^{\prime}\right)\right) \longrightarrow 0 .
$$

Define the submanifolds $Y_{1}:=I \times X^{\prime}$ and $Y_{2}:=Y-\operatorname{Int} Y_{1}=\left(-I \times S^{1} \times I \times\right.$ $\left.S^{1}\right) \cup_{G \times S^{1}}\left(D^{2} \times I \times S^{1}\right)$. Then we see that

$$
Y_{1} \simeq X^{\prime}, Y_{2} \simeq S^{1}, Y_{1} \cap Y_{2} \simeq \partial X^{\prime}=\left(S_{1} \amalg S_{2}\right) \times S^{1} .
$$

By the Meyer-Vietoris exact sequence, we have the exact sequence

$$
\begin{array}{ccc}
H_{2}\left(Y_{1}\right) \oplus H_{2}\left(Y_{2}\right) \longrightarrow H_{2}(Y) \stackrel{\partial_{*}}{\longrightarrow} H_{1}\left(Y_{1} \cap Y_{2}\right) \longrightarrow & H_{1}\left(Y_{1}\right) \oplus H_{1}\left(Y_{2}\right) . \\
H_{2}\left(X^{\prime}\right) \oplus 0 & H_{1}\left(\partial X^{\prime}\right) & H_{1}\left(X^{\prime}\right) \oplus H_{1}\left(S^{1}\right) .
\end{array}
$$

Denote the map $H_{1}\left(\partial X^{\prime}\right) \rightarrow H_{1}\left(X^{\prime}\right) \oplus H_{1}\left(S^{1}\right)$ in the above diagram by $h$. the projection $H_{1}\left(\partial X^{\prime}\right) \rightarrow H_{1}\left(S^{1}\right)$ to the second entry of $h$ is the composite of inclusion homomorphism $H_{1}\left(\partial X^{\prime}\right) \rightarrow H_{1}\left(X^{\prime}\right)$ and $\pi_{*}: H_{1}\left(X^{\prime}\right) \rightarrow H_{1}\left(S^{1}\right)$. Therefore,

$$
\operatorname{Ker}\left(H_{1}\left(\partial X^{\prime}\right) \rightarrow H_{1}\left(X^{\prime}\right) \oplus H_{1}\left(S^{1}\right)\right)=\operatorname{Ker}\left(H_{1}\left(\partial X^{\prime}\right) \rightarrow H_{1}\left(X^{\prime}\right)\right) .
$$

So the sequence is exact.

Next, we will construct the splitting

$$
H_{2}(Y ; \mathbf{Q})=j_{i *} H_{2}\left(X^{\prime} ; \mathbf{Q}\right) \oplus \operatorname{Ker}\left(H_{1}\left(\partial X^{\prime} ; \mathbf{Q}\right) \rightarrow H_{1}\left(X^{\prime} ; \mathbf{Q}\right)\right) .
$$

Note that there exist $p, q \in \mathbf{Q}$ such that

$$
\operatorname{Ker}\left(H_{1}\left(\partial X^{\prime} ; \mathbf{Q}\right) \rightarrow H_{1}\left(X^{\prime} ; \mathbf{Q}\right)\right)=\mathbf{Q}\left(e_{1}+e_{2}\right) \oplus \mathbf{Q}\left\{p\left(e_{3}-e_{4}\right)+q e_{1}\right\}
$$

as in Section 2. To construct the splitting, we choose elements of inverse images of $e_{1}+e_{2}, p\left(e_{3}-e_{4}\right)+q e_{1}$ under $H_{2}(Y) \rightarrow H_{1}\left(\partial X^{\prime}\right)$. Define $\iota_{Y}: \Sigma_{g+1} \rightarrow Y$ by

$$
\begin{aligned}
\Sigma_{g+1} & \rightarrow X \quad \rightarrow I \times X \quad \hookrightarrow Y . \\
x & \mapsto(x, 0) \mapsto(0, x, 0) .
\end{aligned}
$$


By the Meyer-Vietoris exact sequence as above, we have

$$
\begin{gathered}
H_{2}(Y) \rightarrow H_{1}\left(Y_{1} \cap Y_{2}\right) \rightarrow H_{1}\left(\partial X^{\prime}\right), \\
\iota_{Y *}\left[\Sigma_{g+1}\right] \mapsto \partial_{*} \iota_{Y *}\left[\Sigma_{g+1}\right] \mapsto e_{1}+e_{2}
\end{gathered}
$$

so we choose $\iota_{Y *}\left[\Sigma_{g+1}\right]$ as an element of the inverse image of $e_{1}+e_{2}$.

Next, we choose an element of the inverse image of $p\left(e_{3}-e_{4}\right)+q e_{1}$. Since $p\left(e_{3}-e_{4}\right)+$ $q e_{1} \in \operatorname{Ker}\left(H_{1}\left(\partial X^{\prime} ; \mathbf{Q}\right) \rightarrow H_{1}\left(X^{\prime} ; \mathbf{Q}\right)\right)$, there exists a singular 2-chain $s \in C_{2}\left(X^{\prime} ; \mathbf{Q}\right)$ such that

$$
\partial s=p\left(f_{3}-f_{4}\right)+q f_{1} \in B_{1}\left(X^{\prime} ; \mathbf{Q}\right) .
$$

For $i=0,1$, define $s_{0 i}^{\prime}: I \times S^{1} \rightarrow I \times S^{1} \times I \times S^{1} \hookrightarrow Y_{2}$ by $s_{0 i}^{\prime}(t, u)=(i, 0, t, u)$. Then we see that

$$
\left[\partial s_{0 i}^{\prime}\right]=\left[j_{i} f_{3}-j_{i} f_{4}\right] \in H_{1}\left(Y_{1} \cap Y_{2} ; \mathbf{Q}\right) .
$$

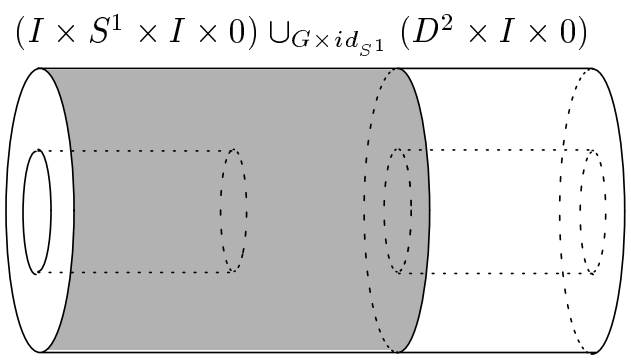

Image of $s_{10}^{\prime}$

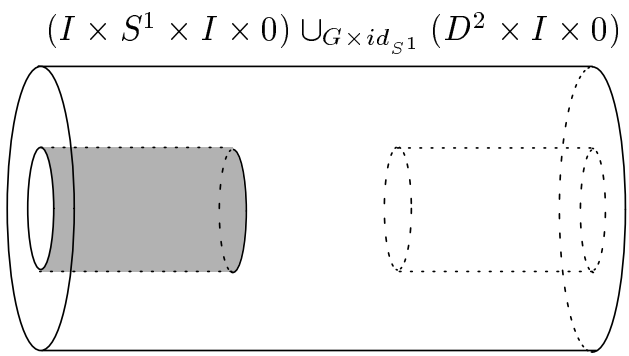

Image of $s_{11}^{\prime}$

Figure 4: Images of $s_{10}^{\prime}$ and $s_{11}^{\prime} \subset Y_{2}$.

Define $s_{1 i}^{\prime}: D^{2} \rightarrow Y_{2}=\left(-I \times S^{1} \times I \times S^{1}\right) \cup_{G \times S^{1}}\left(D^{2} \times I \times S^{1}\right) \subset Y$ as shown in Figure 4 by

$$
\begin{aligned}
& s_{10}^{\prime}(x)=\left\{\begin{array}{lll}
(6 x, 1,0) & \in D^{2} \times I \times S^{1} & \left(\|x\| \leq \frac{1}{6}\right), \\
\left(2-6\|x\|, \frac{x}{\|x\|}, \frac{2}{3}, 0\right) & \in I \times S^{1} \times I \times S^{1} & \left(\frac{1}{6} \leq\|x\| \leq \frac{1}{3}\right), \\
\left(0, \frac{x}{\|x\|}, 1-\|x\|, 0\right) & \in I \times S^{1} \times I \times S^{1} & \left(\frac{1}{3} \leq\|x\| \leq 1\right),
\end{array}\right. \\
& s_{11}^{\prime}(x)=\left\{\begin{array}{lll}
\left(\frac{3}{2} x, 0,0\right) & \in D^{2} \times I \times S^{1} & \left(\|x\| \leq \frac{2}{3}\right), \\
\left(1, \frac{x}{\|x\|}, 1-\|x\|, 0\right) & \in I \times S^{1} \times I \times S^{1} & \left(\frac{2}{3} \leq\|x\| \leq 1\right) .
\end{array}\right.
\end{aligned}
$$

Then, we have $\left[\partial s_{1 i}^{\prime}\right]=\left[j_{i} f_{1}\right] \in H_{1}\left(Y_{1} \cap Y_{2} ; \mathbf{Q}\right)$.

The chain $s_{i}^{\prime}:=p s_{0 i}^{\prime}+q s_{1 i}^{\prime}$ satisfies

$$
\left[\partial s_{i}^{\prime}\right]=\left[j_{i}\left(p\left(f_{3}-f_{4}\right)+q f_{1}\right)\right] \in H_{1}\left(Y_{1} \cap Y_{2} ; \mathbf{Q}\right),
$$


so that we have $\left[\partial\left(j_{i} s-s_{i}^{\prime}\right)\right]=0 \in H_{1}\left(Y_{1} \cap Y_{2} ; \mathbf{Q}\right)$.

We see

$$
\begin{aligned}
& H_{2}(Y ; \mathbf{Q}) \rightarrow H_{1}\left(Y_{1} \cap Y_{2} ; \mathbf{Q}\right) \rightarrow \quad H_{1}\left(\partial X^{\prime} ; \mathbf{Q}\right), \\
& {\left[j_{i} s-s_{i}^{\prime}\right] \mapsto \partial_{*}\left[j_{i} s-s_{i}^{\prime}\right] \quad \mapsto p\left(e_{3}-e_{4}\right)+q e_{1}}
\end{aligned}
$$

so that we can choose $\left[j_{i} s-s_{i}^{\prime}\right]$ as an element of the inverse image of $p\left(e_{3}-e_{4}\right)+q e_{1}$. Now we calculate the intersection form of $H_{2}(Y ; \mathbf{Q})$. Define the subspace $X_{1}^{\prime \prime}=$ $j_{1}(X) \cup_{G \times i d_{S 1}}\left(D^{2} \times 0 \times S^{1}\right) \subset Y$. Then we see that $X_{1}^{\prime \prime}$ is a deformation retract of $Y$. Hence, every element of $H_{2}(Y ; \mathbf{Q})$ is represented by a cycle in $X_{1}^{\prime \prime}$. Therefore, a homology class is included in the annihilator of intersection form in $H_{2}(Y ; \mathbf{Q})$ if it is represented by a cycle which has no common point with $X_{1}^{\prime \prime}$. We see

$$
j_{0}\left(X^{\prime}\right) \cap X_{1}^{\prime \prime}=\varnothing \text { and } \iota_{Y}\left(\Sigma_{g+1}\right) \cap X_{1}^{\prime \prime}=\varnothing,
$$

so that the preimage of $\mathbf{Q}\left(e_{1}+e_{2}\right)$ and $j_{0 *} H_{2}\left(X^{\prime} ; \mathbf{Q}\right)$ are included in the annihilator of intersection form in $\mathrm{H}_{2}(Y ; \mathbf{Q})$.

To describe the signature of $Y$, it suffices to calculate the self-intersection number of $\left[j_{i} s-s_{i}^{\prime}\right]=p\left(e_{3}-e_{4}\right)+q e_{1}$. The cycle $j_{i} s-s_{i}^{\prime}$ satisfies

$$
\begin{array}{r}
\operatorname{Im}\left(j_{0} s\right) \cap\left(\operatorname{Im}\left(j_{1} s\right) \cup \operatorname{Im}\left(s_{01}^{\prime}\right) \cup \operatorname{Im}\left(s_{11}^{\prime}\right)\right)=\varnothing \\
\operatorname{Im}\left(s_{00}^{\prime}\right) \cap\left(\operatorname{Im}\left(j_{1} s\right) \cup \operatorname{Im}\left(s_{01}^{\prime}\right) \cup \operatorname{Im}\left(s_{11}^{\prime}\right)\right)=\varnothing \\
\operatorname{Im}\left(s_{10}^{\prime}\right) \cap\left(\operatorname{Im}\left(j_{1} s\right) \cup \operatorname{Im}\left(s_{11}^{\prime}\right)\right)=\varnothing,
\end{array}
$$

so that

$$
\begin{aligned}
\left(j_{0} s-s_{0}^{\prime}\right) \cdot\left(j_{1} s-s_{1}^{\prime}\right) & =\left(j_{0} s-\left(p s_{00}^{\prime}+q s_{10}^{\prime}\right)\right) \cdot\left(j_{1} s-\left(p s_{01}^{\prime}+q s_{11}^{\prime}\right)\right) \\
& =q s_{10}^{\prime} \cdot p s_{01}^{\prime} .
\end{aligned}
$$

If necessary, perturb the chain $s_{01}^{\prime}$. Then we see that $s_{01}^{\prime}$ and $s_{10}^{\prime}$ intersect only once positively. Hence, we have $\operatorname{Sign}(Y)=\operatorname{sign}(p q)=\operatorname{sign}(m(\varphi))$.

\subsection{Wall's non-additivity formula}

In the introduction, we stated the Novikov additivity of Signature. Wall derives a formula from this additivity in a more general case, when two compact oriented smooth $4 k$-manifolds are glued along common submanifolds of their boundaries. We will give the specific case of his formula for $k=1$.

Let $Z$ be a closed oriented smooth 2-manifold, $X_{-}, X_{0}, X_{+}$compact oriented smooth 3-manifolds with the boundaries $\partial X_{-}=\partial X_{0}=\partial X_{+}=Z$, and $Y_{-}, Y_{+}$ compact oriented smooth 4-manifolds with the boundaries $\partial Y_{-}=X_{-} \cup_{Z}\left(-X_{0}\right)$, 
$\partial Y_{+}=X_{0} \cup_{Z}\left(-X_{+}\right)$. Here we denote by $M \cup_{B}(-N)$ the union of two manifolds $M$ and $N$ glued by orientation reversing diffeomorphism of their common boundaries $\partial M=\partial N=B$. Let $Y=Y_{-} \cup_{X_{0}} Y_{+}$be the union of $Y_{-}$and $Y_{+}$glued along submanifolds $X_{0}$ of their boundaries. Suppose $Y$ is oriented by the induced orientation of $Y_{-}$and $Y_{+}$.

Write $V=H_{1}(Z ; \mathbf{R})$; let $A, B$, and $C$ be the kernels of the maps on first homology induce by the inclusions of $Z$ in $X_{-}, X_{0}$ and $X_{+}$respectively.

We define

$$
W:=\frac{B \cap(C+A)}{(B \cap C)+(B \cap A)},
$$

and a bilinear form $\Psi$ by

$$
\begin{aligned}
\Psi: \quad W \times W & \rightarrow \mathbf{R} . \\
\left(b, b^{\prime}\right) & \mapsto b \cdot c^{\prime} .
\end{aligned}
$$

Here $c^{\prime}$ is an element of $C$ such that there exists an element $a^{\prime} \in A$ such that $a^{\prime}+$ $b^{\prime}+c^{\prime}=0$, and $b \cdot c^{\prime}$ denotes the intersection product of $b$ and $c^{\prime}$. It is known that $\Psi$ is independent of the choice of $c^{\prime}$ and well-defined on $W$. Denote the signature of the bilinear form $\Psi$ by $\operatorname{Sign}(V ; B C A)$ and the signature of the compact oriented 4-manifold $M$ by Sign $M$. We are now ready to state the formula.

\section{Theorem 3.5 (Wall [13]) $\quad \operatorname{Sign} Y=\operatorname{Sign} Y_{-}+\operatorname{Sign} Y_{+}-\operatorname{Sign}(V ; B C A)$.}

\subsection{The differences Sign $E_{g}-\operatorname{Sign} E_{g, 2}$ and Sign $E_{g+1}-\operatorname{Sign} E_{g, 2}$}

In this subsection, we calculate the difference of signature associated with sewing the trivial Disk bundles onto the $\Sigma_{g, 2}$-bundle.

In the introduction, we defined $E_{g, r}^{\varphi, \psi}$ as a oriented $\Sigma_{g, r}$-bundle on $P$ which has monodromies $\varphi, \psi,(\psi \varphi)^{-1} \in \mathcal{M}_{g, r}$ along $\alpha, \beta, \gamma \in \pi_{1}(P)$. If we fix $\varphi, \psi \in \mathcal{M}_{g, 2}$, we denote simply

$$
E_{g, 2}:=E_{g, 2}^{\varphi, \psi}, E_{g}:=E_{g}^{\theta(\varphi), \theta(\psi)} \text {, and } E_{g+1}:=E_{g+1}^{\eta(\varphi), \eta(\psi)}(g \geq 0) .
$$

Proposition 3.6 $\operatorname{Sign}\left(E_{g}\right)-\operatorname{Sign}\left(E_{g, 2}\right)=-\operatorname{sign}\left(m(\varphi)+m(\psi)+m\left((\varphi \psi)^{-1}\right)\right)$ for $g \geq 0$.

Proof $E_{g}$ is the union of $E_{g, 2}$ and $E_{D}:=\left(D^{2} \amalg D^{2}\right) \times P$ glued along their boundaries. Using Non-additivity formula Theorem 3.5, we calculate $\operatorname{Sign}\left(E_{g}\right)-$ $\operatorname{Sign}\left(E_{g, 2}\right)$. 
Define $Y_{-}, Y_{+}, X_{-}, X_{0}, X_{+}$, and $Z$ by

$$
\begin{aligned}
& Y_{-}:=\left(\amalg_{j=1}^{2} D^{2}\right) \times P, \quad Y_{+}:=E_{g, 2}, \\
& X_{-}:=\left(\amalg_{j=1}^{2} D^{2}\right) \times \partial P, \quad X_{+}:=\left.E_{g, 2}\right|_{\partial P}, \quad X_{0}:=\left(\amalg_{j=1}^{2} \partial D^{2}\right) \times P,
\end{aligned}
$$

and $Z:=\left(\amalg_{j=1}^{2} \partial D^{2}\right) \times \partial P$, respectively.

Here, by the notation stated in Section 2.1,

$$
X_{+}=\left.E_{g, 2}\right|_{\partial P} \cong X^{\varphi} \amalg X^{\psi} \amalg X^{(\psi \varphi)^{-1}}, \quad Z \cong \partial X^{\varphi} \amalg \partial X^{\psi} \amalg \partial X^{(\psi \varphi)^{-1}} .
$$

Define $V, A, B$, and $C$ as stated in Section 3.1.

Since $X^{\varphi}=X^{\psi}=X^{(\psi \varphi)^{-1}}=S^{1} \times S^{1}$, we can choose the bases of $H_{1}\left(\partial X^{\varphi} ; \mathbf{R}\right)$, $H_{1}\left(\partial X^{\psi} ; \mathbf{R}\right)$, and $H_{1}\left(\partial X^{(\psi \varphi)^{-1}} ; \mathbf{R}\right)$ as stated in Section 2.1. Denote their bases by $\left\{e_{11}, e_{12}, e_{13}, e_{14}\right\},\left\{e_{21}, e_{22}, e_{23}, e_{24}\right\}$, and $\left\{e_{31}, e_{32}, e_{33}, e_{34}\right\}$ respectively.

Since $Z=\partial X^{\varphi} \amalg \partial X^{\psi} \amalg \partial X^{(\psi \varphi)^{-1}}$, we think of $e_{i j}$ as an element of $H_{1}(Z ; \mathbf{R})$.

Denote $m(\varphi)=\left[a_{1}: b_{1}\right], m(\psi)=\left[a_{2}: b_{2}\right]$, and $m\left((\psi \varphi)^{-1}\right)=\left[a_{3}: b_{3}\right]$ respectively. Then we have

$$
\begin{aligned}
V & =H_{1}(Z, \mathbf{R})=\bigoplus_{i=1}^{3} \bigoplus_{j=1}^{4} \mathbf{R} e_{i j}, \\
A & =\mathbf{R} e_{11} \oplus \mathbf{R} e_{21} \oplus \mathbf{R} e_{31} \oplus \mathbf{R} e_{12} \oplus \mathbf{R} e_{22} \oplus \mathbf{R} e_{32}, \\
B & =\mathbf{R}\left(e_{11}-e_{21}\right) \oplus \mathbf{R}\left(e_{11}-e_{31}\right) \oplus \mathbf{R}\left(e_{12}-e_{22}\right) \oplus \mathbf{R}\left(e_{12}-e_{32}\right) \\
& \oplus \mathbf{R}\left(e_{13}+e_{23}+e_{33}\right) \oplus \mathbf{R}\left(e_{14}+e_{24}+e_{34}\right), \\
C & =\bigoplus_{i=1}^{3} \begin{cases}\mathbf{R}\left(e_{i 1}+e_{i 2}\right) \oplus \mathbf{R}\left(e_{i 3}-e_{i 4}+m_{i} e_{i 1}\right) & \text { if } a_{i} \neq 0 \\
\mathbf{R} e_{i 1} \oplus \mathbf{R} e_{i 2} & \text { if } a_{i}=0 .\end{cases}
\end{aligned}
$$

Here we denote $m_{i}:=\frac{b_{i}}{a_{i}}$. Hence,

$$
B \cap A=\mathbf{R}\left(e_{11}-e_{21}\right) \oplus \mathbf{R}\left(e_{12}-e_{22}\right) \oplus \mathbf{R}\left(e_{11}-e_{31}\right) \oplus \mathbf{R}\left(e_{12}-e_{32}\right),
$$




$$
\begin{aligned}
& B \cap C=\left\{\begin{array}{l}
\mathbf{R}\left(e_{11}-e_{21}+e_{12}-e_{22}\right) \oplus \mathbf{R}\left(e_{11}-e_{31}+e_{12}-e_{32}\right) \oplus \\
\mathbf{R}\left(e_{13}+e_{23}+e_{33}-e_{14}-e_{24}-e_{34}+m_{1} e_{11}+m_{2} e_{21}+m_{3} e_{31}\right) \\
\text { if } a_{i} \neq 0 \text { for } i=1,2,3 \text { and } m_{1}+m_{2}+m_{3}=0, \\
\mathbf{R}\left(e_{11}-e_{21}+e_{12}-e_{22}\right) \oplus \mathbf{R}\left(e_{11}-e_{31}+e_{12}-e_{32}\right) \\
\text { if } a_{i} \neq 0 \text { for } i=1,2,3 \text { and } m_{1}+m_{2}+m_{3} \neq 0, \\
\mathbf{R}\left(e_{11}-e_{21}+e_{12}-e_{22}\right) \oplus \mathbf{R}\left(e_{11}-e_{31}+e_{12}-e_{32}\right) \\
\text { if } a_{1}=0, a_{2} \neq 0, a_{3} \neq 0, \\
\mathbf{R}\left(e_{11}-e_{21}\right) \oplus \mathbf{R}\left(e_{12}-e_{22}\right) \oplus \mathbf{R}\left(e_{11}-e_{31}+e_{12}-e_{32}\right) \\
\text { if } a_{1}=a_{2}=0, a_{3} \neq 0, \\
\mathbf{R}\left(e_{11}-e_{21}\right) \oplus \mathbf{R}\left(e_{12}-e_{22}\right) \oplus \mathbf{R}\left(e_{11}-e_{31}\right) \oplus \mathbf{R}\left(e_{12}-e_{32}\right) \\
\text { if } a_{i}=0 \text { for } i=1,2,3,
\end{array}\right. \\
& B \cap(C+A)=\left\{\begin{array}{l}
\mathbf{R}\left(e_{11}-e_{21}\right) \oplus \mathbf{R}\left(e_{12}-e_{22}\right) \oplus \mathbf{R}\left(e_{11}-e_{31}\right) \oplus \mathbf{R}\left(e_{12}-e_{32}\right) \\
\oplus \mathbf{R}\left(e_{13}+e_{23}+e_{33}-e_{14}-e_{24}-e_{34}\right) \\
\text { if } a_{i} \neq 0 \text { for } i=1,2,3 \\
\mathbf{R}\left(e_{11}-e_{21}\right) \oplus \mathbf{R}\left(e_{12}-e_{22}\right) \oplus \mathbf{R}\left(e_{11}-e_{31}\right) \oplus \mathbf{R}\left(e_{12}-e_{32}\right) \\
\text { otherwise. }
\end{array}\right.
\end{aligned}
$$

By computing the signature of $\Psi$, we have

$$
\operatorname{Sign}(V ; B C A)=\left\{\begin{array}{lr}
\operatorname{sign}\left(m_{1}+m_{2}+m_{3}\right) \text { if } a_{i} \neq 0 \text { for } i=1,2,3 \\
0 & \text { otherwise }
\end{array}\right.
$$

For example, consider the case when $a_{i} \neq 0$ for $i=1,2,3$ and $m_{1}+m_{2}+m_{3} \neq 0$. Then, the space $W$ is generated by the element represented by

$$
b:=e_{13}+e_{23}+e_{33}-e_{14}-e_{24}-e_{34} \in B \cap(C+A) .
$$

Choose the elements

$$
a:=m_{1} e_{11}+m_{2} e_{21}+m_{3} e_{31} \in A \text { and } c:=-\sum_{i=1}^{3}\left(e_{i 3}-e_{i 4}+m_{i} e_{i 1}\right) \in C .
$$

Then we see that $a+b+c=0$ and obtain $\Psi(b, b)=b \cdot c=m_{1}+m_{2}+m_{3}$. This shows that $\operatorname{Sign}(V ; B C A)=\operatorname{sign}\left(m_{1}+m_{2}+m_{3}\right)$. The other cases follow in similar ways.

Hence, we obtain

$$
\operatorname{Sign}(V ; B C A)=\operatorname{sign}\left(m(\varphi)+m(\psi)+m\left((\varphi \psi)^{-1}\right)\right) .
$$


By the non-additivity formula, we have

$$
\operatorname{Sign}\left(E_{g}\right)=\operatorname{Sign}\left(E_{D}\right)+\operatorname{Sign}\left(E_{g, 2}\right)-\operatorname{Sign}(V ; B C A) .
$$

Since $E_{D}$ is a trivial bundle $\left(D^{2} \amalg D^{2}\right) \times P$, we have $\operatorname{Sign}\left(E_{D}\right)=0$.

This completes the proof of the proposition.

By Theorem 3.1 and Proposition 3.6, we can calculate the difference of signature $\operatorname{Sign}\left(E_{g}\right)-\operatorname{Sign}\left(E_{g, 2}\right)$.

Corollary 3.7 For $g \geq 0$,

$$
\begin{aligned}
\operatorname{Sign}\left(E_{g+1}\right)-\operatorname{Sign}\left(E_{g, 2}\right)= & \operatorname{sign}(m(\varphi))+\operatorname{sign}(m(\psi))+\operatorname{sign}\left(m\left((\varphi \psi)^{-1}\right)\right) \\
& -\operatorname{sign}\left(m(\varphi)+m(\psi)+m\left((\varphi \psi)^{-1}\right)\right) .
\end{aligned}
$$

\section{References}

[1] M Atiyah, The logarithm of the Dedekind $\eta$-function, Math. Ann. 278 (1987) 335-380 MR909232

[2] H Endo, Meyer's signature cocycle and hyperelliptic fibrations, Math. Ann. 316 (2000) 237-257 MR1741270

[3] J L Harer, The second homology group of the mapping class group of an orientable surface, Invent. Math. 72 (1983) 221-239 MR700769

[4] J L Harer, Stability of the homology of the mapping class groups of orientable surfaces, Ann. of Math. (2) 121 (1985) 215-249 MR786348

[5] S Iida, Adiabatic limit of $\eta$-invariants and the Meyer function of genus two, Master's thesis, University of Tokyo (2004)

[6] R Kasagawa, On a function on the mapping class group of a surface of genus 2, Topology Appl. 102 (2000) 219-237 MR1745445

[7] N Kawazumi, A generalization of the Morita-Mumford classes to extended mapping class groups for surfaces, Invent. Math. 131 (1998) 137-149 MR1489896

[8] M Korkmaz, A I Stipsicz, The second homology groups of mapping class groups of oriented surfaces, Math. Proc. Cambridge Philos. Soc. 134 (2003) 479-489 MR1981213

[9] W Meyer, Die Signatur von Flächenbündeln, Math. Ann. 201 (1973) 239-264 MR0331382

[10] J W Milnor, J D Stasheff, Characteristic classes, Annals of Mathematics Studies 76, Princeton University Press (1974) MR0440554

[11] T Morifuji, On Meyer's function of hyperelliptic mapping class groups, J. Math. Soc. Japan 55 (2003) 117-129 MR1939188 
[12] J Powell, Two theorems on the mapping class group of a surface, Proc. Amer. Math. Soc. 68 (1978) 347-350 MR0494115

[13] C T C Wall, Non-additivity of the signature, Invent. Math. 7 (1969) 269-274 MR0246311

Graduate School of Mathematical Sciences, The University of Tokyo 3-8-1 Komaba Meguro-ku, Tokyo, 153-8914, Japan sato@ms.u-tokyo.ac.jp

Received: 20 February 2008 Revised: 30 May 2008 